\title{
A New Cosmological Model for Matter, Energy, Sound, the Origin of the Universe and Gravity
}

\author{
Madonna-Megara Holloway \\ Acumen Consulting \\ 205 Westridge Lane \\ Anmore, BC, Canada, V3H $0 A 3$ \\ 604-469-9446; madonna-megara@madonna-megara.com
}

\begin{abstract}
This paper is an abstract of a greater volume of work more than 30 years in formation. It is a thought experiment. In order to present certain concepts, statements are made without supporting scientific observations which would require a substantial manuscript to present. This paper defines a new fabric for space and hypothesizes that matter is formed from this fabric through the agency of Black Holes. Dark sound is theorized. As a consequence of this hypothesis a new model for the origin of the universe is given including new definitions for Black Holes, Dark Matter, and Dark Energy. Neutral electricity, cosmic planes and sub planes are defined. New processes for the coalescing of atoms, the rotation of planets and the observed phenomena of Cosmic Fire are given. Gravity is defined as the result of magnetism and chemical bonds between structures.
\end{abstract}

Keywords: Standard Model, Transport Medium, Gravity, Matter, Energy, Space, Big Bang, Electromagnetic Spectrum, Dark Sound, Dark Matter, Dark Energy, Totusmateria, Bach's Fugue, Resonance Frequency, Hypergammic Radiation, Universe, Origin, Atoms, Fohat, Sonoluminescence

PACS: $95.35+\mathrm{d}$; 95.36. +x; 98.80.-k; 98.80.Cq; 98.80.Bp; 98.80.Ft

\section{INTRODUCTION}

The concepts in this paper have been mostly extracted from two large bodies of work; A Treatise on Cosmic Fire (Bailey, 1973) and The Secret Doctrine (Blavatsky, 1977) with other portions from works by the same authors (Bailey, 1962). It is intended as a bridging paper between esoteric philosophy fact and scientific hypothesis. The concepts have been translated into the language of physics and chemistry from the often obtuse language found in philosophy. It should be noted that not everything that is written in a scientific journal is true but just because a thought comes from an expected direction doesn't mean it is wrong.

\section{Law of Correspondence or Analogy}

Plato and Aristotle posited the concept of shared abstraction where objects did not necessarily share a relation in the usual sense but also a pattern, a regularity, an attribute, an effect or an object. Recurrence relationships demonstrate the pattern forming occurrences demonstrated in the microcosm. By the use of the Law of Analogy, the following demonstrates the means of which the microcosm can arrive at a glimmer of understanding of the more obvious aspects of the macrocosm or vice versa. There is an essential similarity which a science of comparative analysis can be utilized to understand the micro/macro processes. Comparative analysis or analogy is the key which unlocks the understanding and is an interpretive agency; however, the analogy is never exact in detail but only in certain broad basic correspondences.

One of the postulates of this paper is that the Earth is anomalous in the universe. Whether or not it is replicated somewhere else is not important. What is important is that analogous processes, dimensions, actions and reactions found in the universe are replicated on this planet in some form. That the process may demonstrate differently due to the phasing of the environment or that processes which are negligible in this environment become of profound 
importance in the cosmos is also true but there is an essential similarity. With this postulate, analogy will be used throughout to demonstrate a new scientific model for the Nature of Everything or the NOE Hypothesis (pronounced like the word 'know').

\section{FUNDAMENTALS}

"It is impossible, when the complicated facts of an entirely unfamiliar science are being presented to untrained minds for the first time, to put them forward with all their appropriate qualifications.........We must be content to take broad rules first and deal with the exceptions afterwards...." (Blavatsky, 1977)

\section{Totusmateria}

The NOE Hypothesis is a paradigm shift in how science can view the physical world about us. It can be thought of as an alternative explanation for space, matter and energy from that of the Standard Model. Because of the scope of the subject matter, this paper cannot cover the entire breadth of this hypothesis nor go into the depth of detail that many of its tenets deserve. The original works cover several thousand pages. Instead, this paper will only describe the highest level points relative to this subject so that the some impacts of the hypothesis may be appreciated and should allow researchers a basis upon which to formulate further investigation into its veracity.

The NOE Hypothesis starts by stipulating that there exists an essence that defines all of space. This essence is called totusmateria in the NOE Hypothesis, meaning that it is the total of all material in the universe - it is ALL. Totusmateria as a cosmic substance, it is the root of matter; it contains the cosmic genes for differentiation and replication. It has its own properties which we observe and usually assign to other causes until experimentation proves the theory incorrect. Totusmateria comes in various forms the two most notable are differentiated matter that which has form and undifferentiated matter - that which has no form and is part of the root. Matter is indestructible therefore totusmateria equals the sum of all forms. The properties of totusmateria overlays all observed phenomenon. In fact, it will be shown later in this paper that the only thing that truly exists relative to matter and form in this universe are sound waves, totusmateria and energy.

As these three are examined closer it will become apparent the importance of the interaction of these three and the artifacts that they demonstrate.

\section{Dark Sound and Planes}

Physicists have recently theorized Dark Matter and Dark Energy and have through observation substantiated their existence. Despite the efforts Dr Andrew Beckwith has stated in numerous papers the problems associated with the standard model: "If the relative entropy of $10^{89}$ is roughly equivalent to the sum total of one million or so black holes in the center of one million spiral galaxies, then it may remove the necessity of embedding the universe in a larger super structure. If on the other hand, there is a radical difference in the summed value, this difference may be a smoking gun indicating either the necessity of higher dimensions than four for our universe's space time, or that the metrics for curved space time used to compute event horizons are seriously flawed and need additional work." (Beckwith, 2009) That is to say - something is just wrong - it doesn't add up and a radical change is needed; a change which must be supported by empirical data but not necessarily models.

The hypothesis presented in this paper suggests that there is more to sound than what is determined by the physiology of the human ear or what is detected by classical instrumentation. As stated above there is sound, matter and energy - both known and dark.

The entire universe is totusmateria with sound waves and energy rippling through it. As demonstrated in Figure 1, these waves are perceived in the four known dimensions (but more speculated) as electromagnetic radiation occupying a spectrum that appears below. It is important to note that the NOE Hypothesis suggests that the EM spectrum is actually just various forms of a sound wave with the phenomena of light being a sound wave where the matter is rotating in reverse direction to the plane. As the sound wave increases in frequency scientific 
measurements clearly indicate that at the bottom end of the spectrum, or radio waves, behaves more like waves but as frequency increases particle properties become more pronounced.

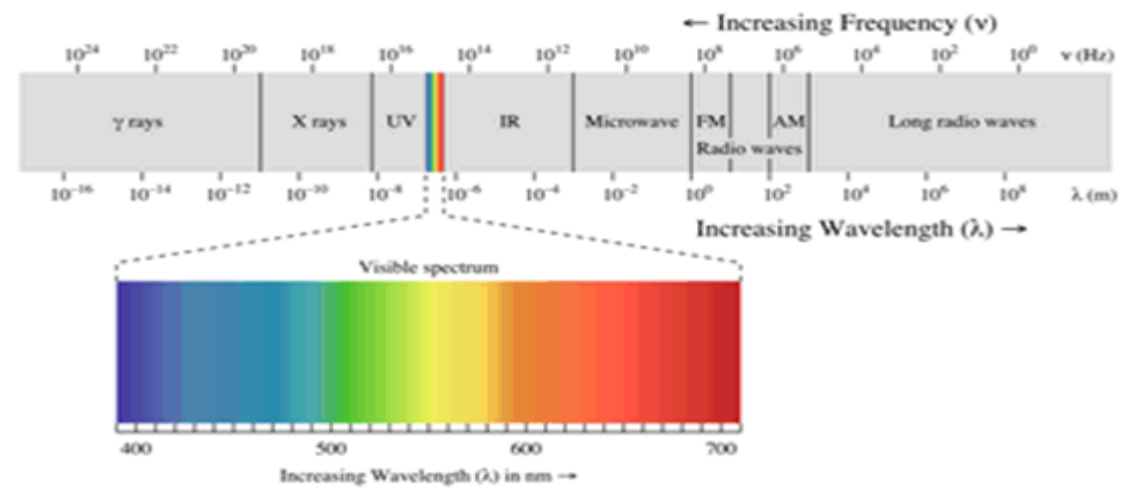

Figure 1. The Electromagnetic Spectrum.

There is another version that is theorized as residing on six more planes or layers with each plane corresponding to a tone giving a total of 7 layers; A through G. An analogy is the 7 defined layers of the planet - solid, water, troposphere, stratosphere, mesosphere, thermosphere and exosphere. The observed EM band is analogous to the physical or the solid layer of the planet. Therefore, all of our observations are amongst the rocks. For the lack of a better term the EM bands on other planes is referred to as Dark Sound. Dark Sound is carried in the $5^{\text {th }}$ dimension and has similar properties to the known EM band. As dark sound increases in frequency dark particle properties become more pronounced. Further, there is a form of light which resides on other planes. As observed in the current Earth analogy, sound on other planes affects the lower planes. That is a sonic boom in air affects water and rocks. This paper refers to sound on all planes as just SOUND. The concept is demonstrated in Figure 2.

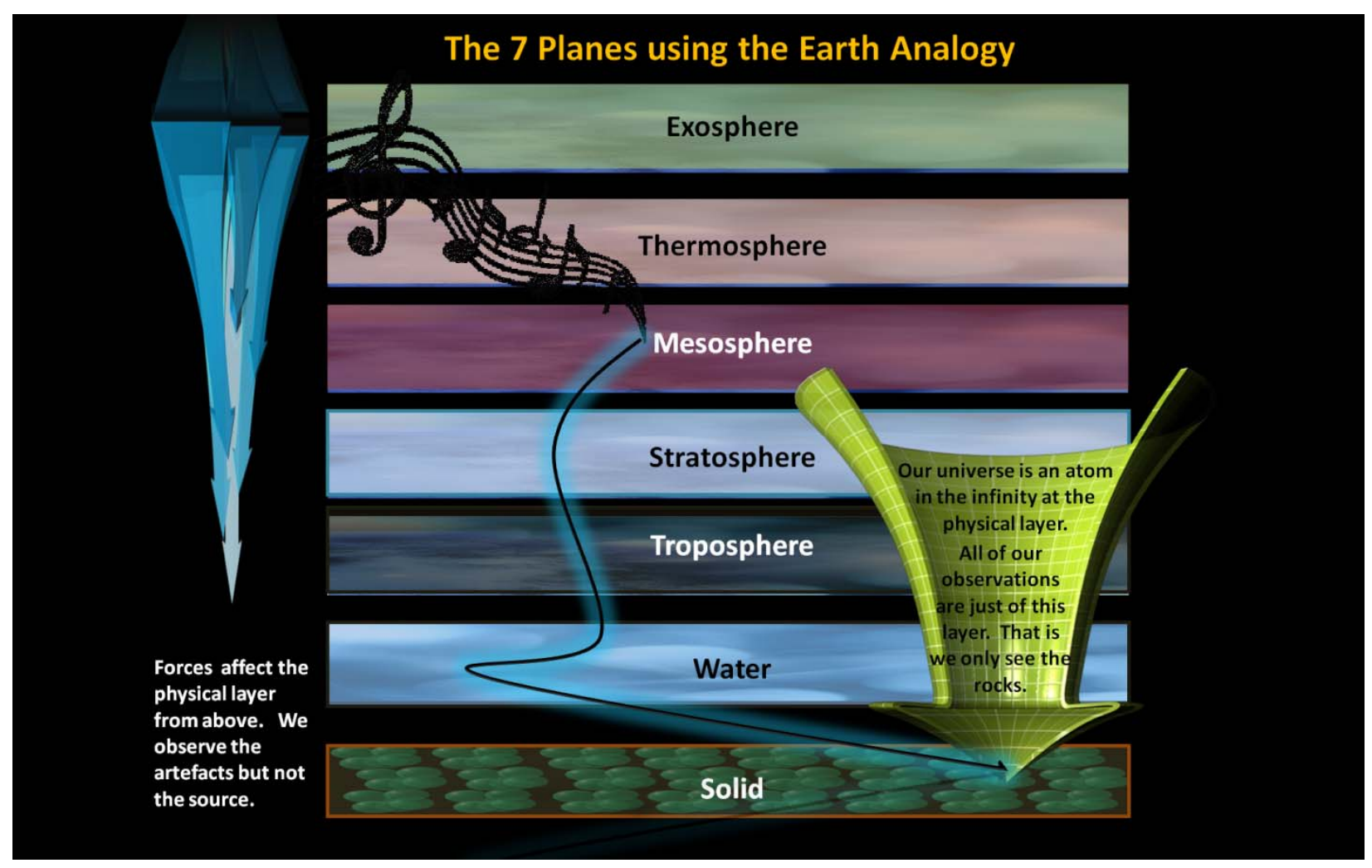

Figure 2. The 7 Planes Using the Earth Analogy 
The earth analogy expands so that the hypothesis assumes that each plane has 7 sub planes. In a solar system analogy there are 49 planes from the Sun's core to the Earth's core. For example the first plane is the Sun with the following sub planes 1.Inner Core; 2.Radiative Zone; 3.Convection Zone; 4.Subsurface Flow; 5.Photosphere; 6.Chromosphere and 7.Corona. The bottom plane is the dense physical Earth with the following sub planes: 7.Lithosphere; 6.Upper Solid; 5.Asthenosphere; 4.Upper Mantle; 3.Lower Mantle; 2Outer Core; and 1. Inner Core. There is a correspondence between the cores of the Sun and the Earth.

\section{The Continuation of the EM Band}

When considering electromagnetic (EM) radiation through space two factors are noted: the speed of transmission of EM radiation through space (i.e. the speed of light, $c$ ) and the upper limit of the EM spectrum (i.e. the highest measured energy of isotropic gamma radiation at approximately $200 \mathrm{MeV}$ (Thompson and Fitchel, 1982) or $4.8 \times 10^{22} \mathrm{H}_{\mathrm{z}}$ ). There have been momentary point flashes of gamma radiation detected above this level (see: http://en.wikipedia.org/wiki/Ultra-high-energy_cosmic_ray) but they are not continuous and emanating from all directions.

These two properties could suggest that a transport medium is present and that the medium not only determines the speed at which EM transfers through it but that something occurs once the frequency of the radiation reaches approx. $4.8 \times 10^{22} \mathrm{H}_{\mathrm{z}}$.

Further, it is demonstrated that the rest mass of a proton ( $\mathrm{an} \mathrm{H}^{1}$ atom) is known to be approx. 1.007276 atomic mass units (amu) (Nave, 2001). Expressed in terms of mass that becomes approx. $1.67363 \times 10^{-27} \mathrm{~kg}$ $\left(1 \mathrm{amu}=1.66054 \times 10^{-27} \mathrm{~kg}\right)$. Einstein's equation that converts mass into energy is:

$$
E=m c^{2}
$$

Using this equation one gets the approximate energy of a proton to be $1.50418 \times 10^{-10}$ Joules. Planck's equation that converts quantum frequency into energy is:

$$
E=h v
$$

Substituting in the energy of a proton for $E$ in this equation one gets the approximate frequency of a proton to be $2.27012 \times 10^{23} \mathrm{H}_{\mathrm{z}}$. The interesting coincidence is the fact that this frequency is only a factor of 4.7 times the upper limit of isotropic gamma radiation. In other words, the electromagnetic spectrum spans 22 orders of magnitude from DC up to its upper limit and then within less than half a magnitude above that the frequency equivalence of the first element appears. But in order to raise this interesting coincidence up to an intriguing coincidence, one only needs to convert the frequency into a wavelength:

$$
\lambda=c / v
$$

The wavelength of a proton turns out to be $1.32060 \mathrm{fm}$. But the equation that approximates the nuclear radius of an atom based on its mass number $\mathrm{A}$ is:

$$
R=r_{0} A^{1 / 3} f m
$$

Where $R$ is the nuclear radius and $r_{0}=1.2$. For a single proton this yields an approximate radius of $1.2 \mathrm{fm}$, a difference of only $10 \%$. In other words, there appears to be a correlation between the mass and radius of a proton and the upper limit of the electromagnetic spectrum.

Is it possible that the constitution of a proton is, in fact, simply electromagnetic radiation at energy levels above that of gamma radiation, in an upper band of (for lack of a better term) Hypergammic radiation? The hypotheses states yes. That is, through processes described below atoms coalesce from totusmateria and represent the continuation of the EM band. The first atom in the periodic table and hence the first in the continuation of the EM band is hydrogen.

\section{Production of Hydrogen through Radio Waves in Salt Water}

John Kanzius (see: http://en.wikipedia.org/wiki/John_Kanzius) in an attempt to find a cure for cancer stumbled upon an oddity of thermodynamics when he accidentally applied a radio wave to salt water which apparently caused 
hydrogen and oxygen to be released. The Kanzius experiment was later replicated by Roy (see: http://en.wikipedia.org/wiki/Rustum_Roy) a materials scientist at Pennsylvania State University. According to Roy, "The radio frequencies act to weaken the bonds between the elements that make up salt water, releasing the hydrogen. Once ignited, the hydrogen will burn as long as it is exposed to the frequencies. The temperature and flame color varies with water solutions and concentrations." If by analogy totusmateria is equivalent to the salt water, then the coalescing of hydrogen from totusmateria through the application of a sound wave, is by analogy demonstrated.

\section{Sonoluminescence}

Frenzel and Schultes (see: http://en.wikipedia.org/wiki/Sonoluminescence) accidentally stumbled upon an oddity of sonar when they applied an ultrasound transducer in a tank of photographic developer fluid. They observed that the bubbles were emitting light. Later, in 1984, Gaitan and Crum (see: http://en.wikipedia.org/wiki/Sonoluminescence) where able to produce a single-bubble, sonoluminescence, event. That is that as long as the sound wave is present in

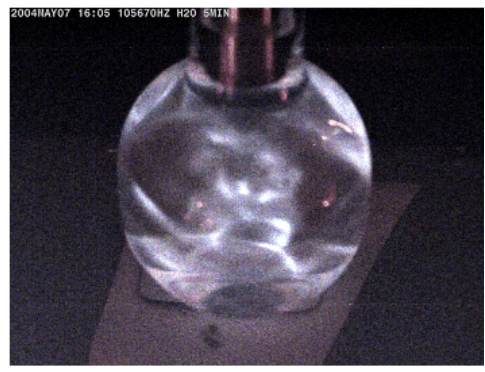

Figure 3. Sonoluminescence. a stable environment a pulse of light is observed. The phenomenon is reportedly not well understood with such exotic explanations as Hawking radiation, virtual particles, black-body radiation, plasma ionization, quantum vacuum fluctuations, or coherent optical lasing etc.

Wrbanek, Fralick, and Wrbanek (2007) demonstrated in their research paper; high temperatures and kinetic energy with the possibility of fusion reaction. The Figure 3 shows a true color picture of sonoluminescence in light water (contrast enhanced) Exposure time was 2 min. at f/2.8 (Page 6). Comparative analysis demonstrates the apparent similarity between sonoluminescence and the observations of supernovae and gases in the macrocosm. That is to say that a single event of sonoluminescence is by analogy a 'supernova in a beaker'.

\section{Summary}

The components discussed above appear to be fragments, parts of a puzzle which need to be put together to produce the whole. That is if you take the concept of the root of matter, with 7 planes each with its own EM spectrum which continues into the periodic table, and apply sound waves a model develops. This model is discussed below. As in all models it is important that empirical data fit. It is not important that established models with known issues such as the Big Bang are accounted for.

\section{THE ORIGIN OF THE UNIVERSE}

In the beginning there was primordial, undifferentiated, pregenetic cosmic substance or totusmateria. This substance was tainted from the previous cycles of the great wave that the hypothesis calls Bach's Fugue after Johannes Sebastian Bach and the mathematical genius of his music (Pedersen, 2009). Fugues are typically composed of three separate contrapuntal voices with 7 notes per voice given a total of 21 notes plus the sub tones combined into one. The hypothesis suggests that the great wave which sounded and created the universe comes in three parts with each part corresponding to three states of electrical energy - the observed and commonly used negative electricity which powers our lives, positive electricity represented by the release of energy when the atom is split, and a neutral form of electricity with properties that synthesize, blend and equilibrate the other two forms. This form although observed is not recognized as yet but controls the spheroidal form of the atom both microscopic as in hydrogen and macroscopic as in galaxies. This sound wave travels in the $5^{\text {th }}$ dimension (not including time).

The exact form of Bach's Fugue is not known at this time and can only be speculated but it is assumed that the composition of the wave is exceedingly complex. The notion that the universe can be modeled after tones or notes was delineated as early as Pythagoras. Although mired in historical controversy (see, http://plato.stanford.edu/ entries/pythagoras/), Pythagoras through observation concluded that "the heavenly bodies also appear to move in accordance with the mathematical ratios that govern the concordant musical intervals in order to produce music of 
the heavens..." The hypothesis states that Pythagoras was correct. Bach demonstrated through his fugues that without mathematics there is no music (Pedersen, 2009). Therefore the universe can only be properly modeled through numbers, sound and color where a tenet of the hypothesis is that these three are synonymous terms. Clifton Callender at Florida State University, Ian Quinn at Yale University and Dmitri Tymoczko at Princeton University devised a method of analyzing complex mathematics into geometric forms as shown below. This form of

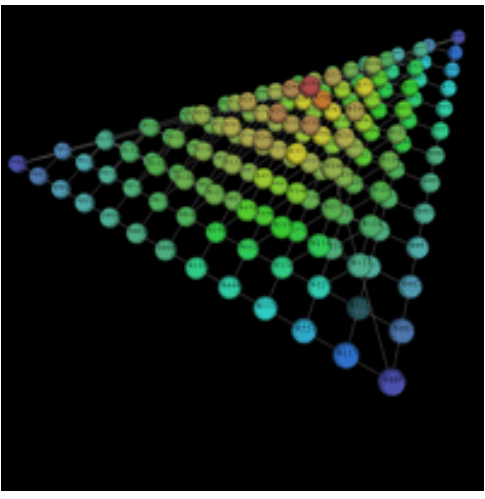

Figure 4. Complex Mathematics in Geometric Form mathematics will eventual find the 'composition' of Bach's Fugue and the beginning of the universe to the current condition will be determined.

"The figure (Figure 4) shows how geometrical music theory represents fournote chord-types - the collections of notes from a tetrahedron, with the colors indicating the spacing between the individual notes in a sequence. In the blue spheres, the notes are clustered, in the warmer colors, they are farther apart. The red ball at the top of the pyramid is the diminished seventh chord, a popular 19th-century chord. Near it are all the most familiar chords of Western music." (Callender, Quinn, and Tymoczko, 2008)

The use of recurrence relations of which the Fibonacci sequence is a linear example and fractal theory will help explain the formation of the various forms from a short chain hydrocarbon to the complex nature of a galactic atom such as the Milky Way. There is a direct correlation between the tones and rhythm of sound and the formation of forms in matter which follow the recurrence relation.

The hypothesis suggest that instead of a 'big bang' being the genesis of the known universe that a complex sound wave in three parts within totusmateria allowed atoms, planets, stars, and galaxies to coalesce. This phenomenon continues.

This also suggests that the total amount of observed physical matter in the universe is not a constant and as analogous to energy where total energy is equal to kinetic energy plus potential energy; total matter is equal to actual matter plus potential matter as stored in totusmateria. In other words the universe did not expand from a singularity but simply condensed out from the root of matter.

\section{Black Holes}

The mechanism which produces this phenomenon is the Black Hole where sound, matter and energy come together in a beautifully synchronous fashion and is illustrated below. It should be noted that totusmateria is not a principle and only responds to the other two. It is the cosmic gene pool from which form is made but only when energy and sound act upon it. It remains even when the other two have ceased. Totusmateria contains the code for differentiation and replication.

The actual black hole is a vortex of inconceivable rapidity of sound which carries the three forms of electricity as defined below:

1. Positive or synthesizing electricity which is understood as the core of the atom and demonstrated through a nuclear blast. It is characterized as a tendency to concentrate at a center or merge.

2. Equilibrating, balancing or neutral electricity which includes chemical affinity and gravity and holds together the form. It is characterized as a pull and is analogous to magnetism and forces associated with chemical bonding.

3. Negative electricity as understood by the electron and is characterized by an urge to:

a. Vibrate

b. Adapt or to rotary movement

c. Repulse or non-attach and also keeps the atom rotating at fixed points

d. Friction or the heat aspect.

As noted in Figure 5 below the vortex is encased in a film or membrane which is not the event horizon - it is actual matter. The origin of the sound wave is the $7^{\text {th }}$ plane counting up from the physical and in the $5^{\text {th }}$ dimension. There is a massive black hole for the universe itself, and millions upon hundreds of millions of lesser black holes which are 
at the center of every atom, system, and galaxy. The location, quality, tone and other factors are controlled by Bach's Fugue as it plays out the rhythm.

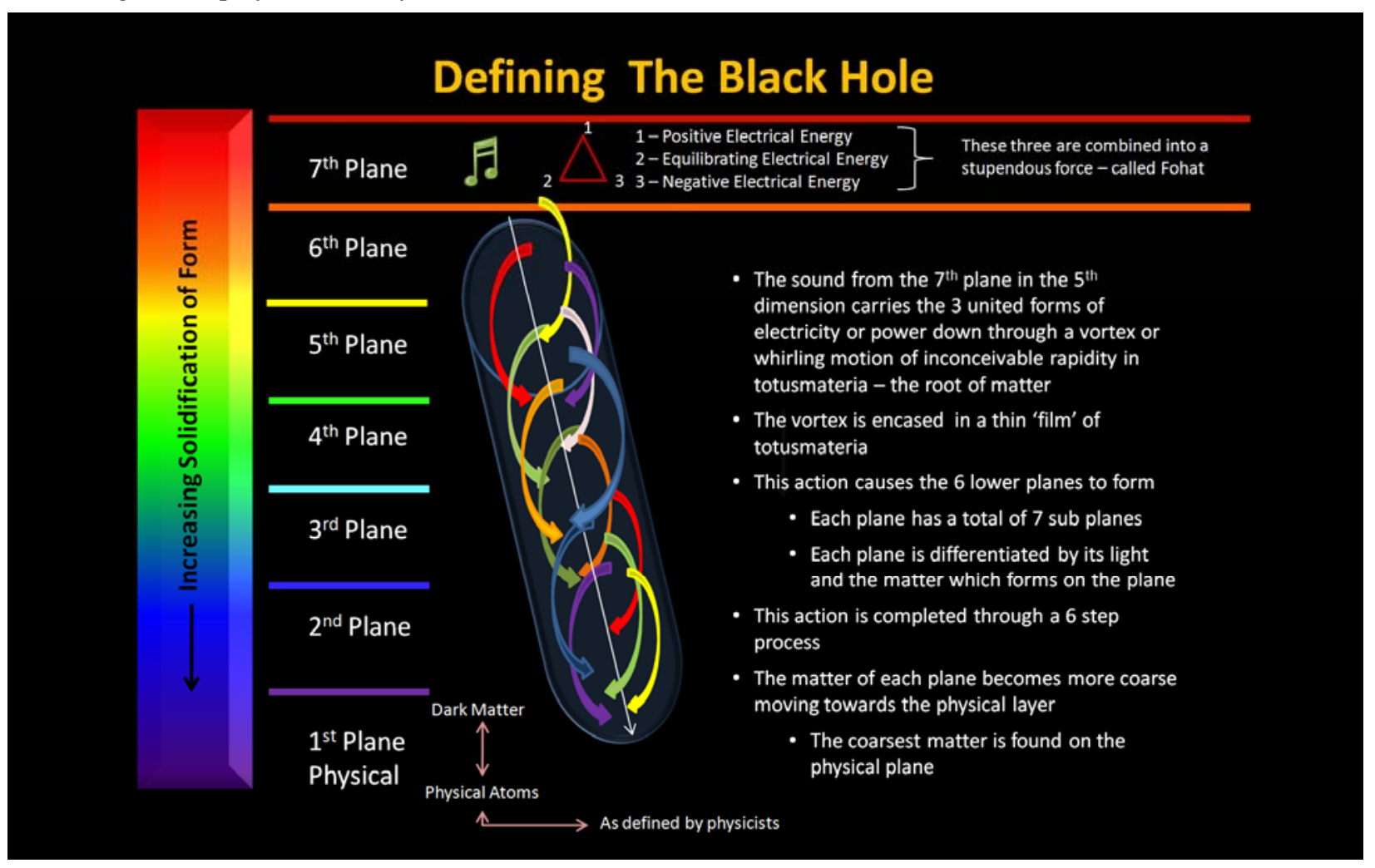

Figure 5. Defining the Black Hole.

The coalescing of the atom proceeds in six stages (a lower frequency of sound in denser material has the same steps):

Stage 1 - This stage is characterized by the first hint of differentiation and assumes a fine misty aspect.

Stage 2 - Condensation begins but is very chaotic and there is no form. The beginnings of excessive heat and violent activity characterize this stage. Inchoate rotary action begins.

Stage 3 - The sphere of the atom begins to form as it responds to the sound wave. Rotary action becomes more coherent and hence heat increases substantially.

Stage 4 -The sphere of what is becoming the atom becomes more condensed and appears to go through a liquefaction stage. The outer surface begins to solidify, heat increases at the centre core or nucleus and the pulsation of the atom starts.

Stage 5 - The atom proceeds to synthesize and the rhythm of the atom to the tone is established. The coloring and vibratory response of the atom can be seen. All parts of the atom are now moving to the chord of the tone in three parts:

- The nucleus responds to part 1 - positive charge.

- The spheroidal form responds to part 2 - synthesizing or equilibrating.

- The electrons respond to part 3 - negative charge.

This stage is very important as the synthesis and synchronization of the positive and negative charges and the equilibrating of the spheroidal form determines the quality, appearance, attributes, and properties of the atom.

Stage 6 - This final stage is the completed atom which now appears to be substance and matter as partly understood by classical chemistry and physics. The nucleus is rotating and the vortex about the axis is clearly defined. The 
cohesiveness is only maintained as long as sound is present. If the sound wave stops then dissolution occurs and the differentiated atom returns to undifferentiated totusmateria.

The sound wave determines the form which has coalesced using the cosmic genetic code stored in totusmateria.

Another way of viewing the process, an analogy, is in terms of biological reproduction:

- The male aspect or father is represented by the black hole. It contributes the electricity for animation, the tone, quality and color of the form which appears.

- The female aspect or mother is represented by totusmateria. It contributes the matter and the cosmic code for differentiation and replication.

- The offspring, created by merging the male and female aspects, is all forms.

\section{Dark Matter and Energy}

The Figure 5 also demonstrates the significance of the photoelectric effect where blue light creates a current but red light will not. The $7^{\text {th }}$ plane has no particle properties and solidification of form increases as the physical layer is approached towards the color of violet. That is to say, the planes are replicated in the visible light spectrum. Further evidence of this is the observed phenomena of Dark Matter being of a finer grade substance on a higher plane within the cosmic physical plane as noted below. Please see Figure 6 and Figure 7 which shows a fourth etheric system.

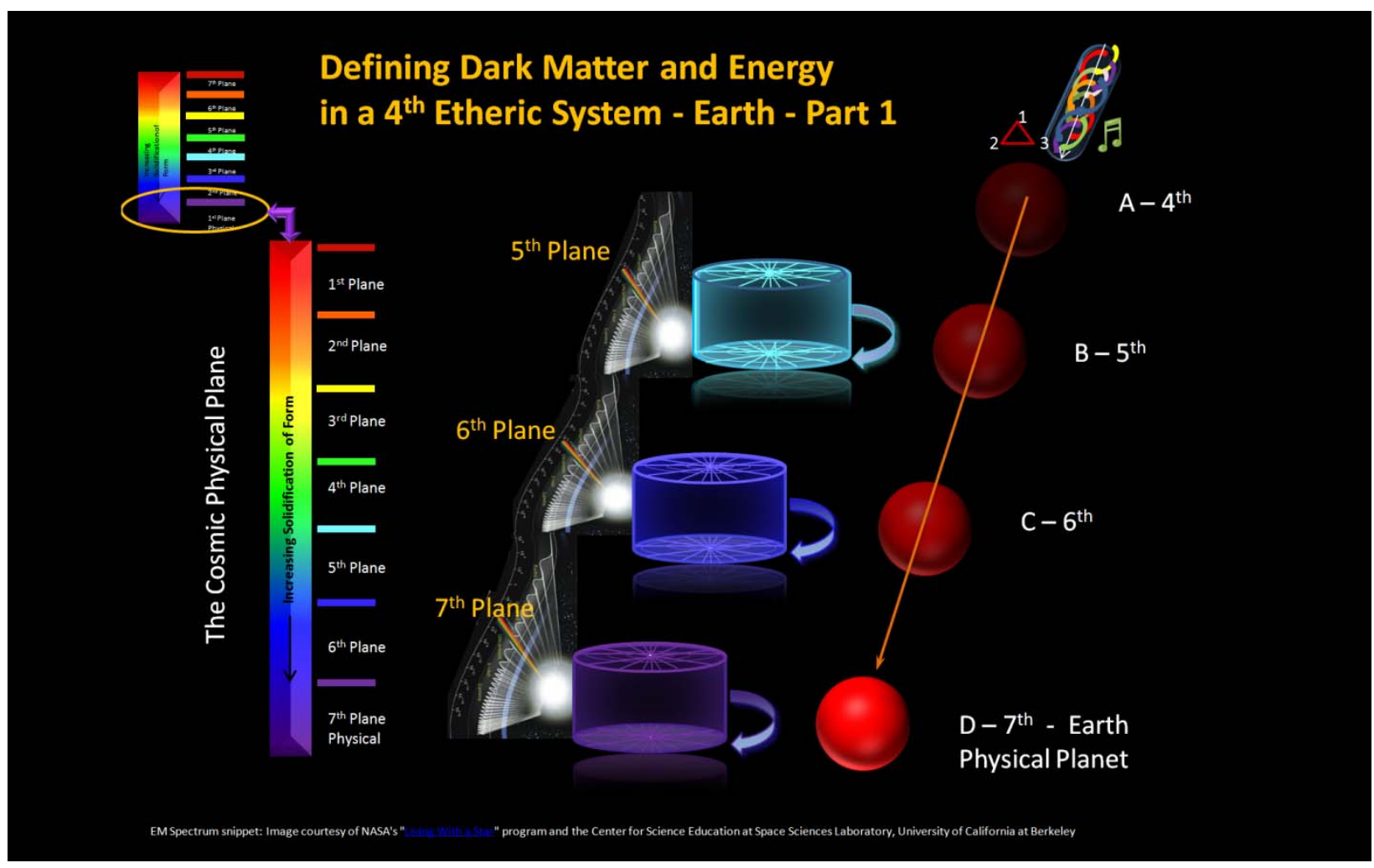

Figure 6. Defining Dark Matter and Energy in a $4^{\text {th }}$ Etheric System - Earth - Part 1 (EM Spectrum snippet: Image courtesy of NASA's "Living With a Star" program (http://ds9.ssl.berkeley.edu/LWS_GEMS/index.htm) and the Center for Science Education at Space Sciences Laboratory, University of California at Berkeley).

Figure 6 shows the solidification of planet Earth from the $4^{\text {th }}$ Etheric plane as it coalesces to the physical plane. Planes 5, 6 and 7 are the material planes were matter is the coarsest and hence detected. Ether and matter are synonymous terms but graduated according to solidification. Ether is undifferentiated totusmateria; matter is differentiated by fohat carried by sound in the $5^{\text {th }}$ dimension. Not shown are the ascending arc and the transition of the planet as it returns to the higher planes. This phenomenon accounts for the missing mass. 
The Earth from its perspective is the nucleus and carries with it the electrons and other particles as defined by chemistry for atoms. As Earth condenses from totusmateria it develops its molecular structure as noted in Figure 7. Only Earth sits in the visible plane. All other globes sit in Dark Matter. The atom is encased in an ovoid of neutral electricity. The chemical bonds are noted in bluish colors and the connection to the surrounding schemes of Venus, Mercury etc. are also shown. Earth is bonded to the Sun as an electron and is discussed later under gravity

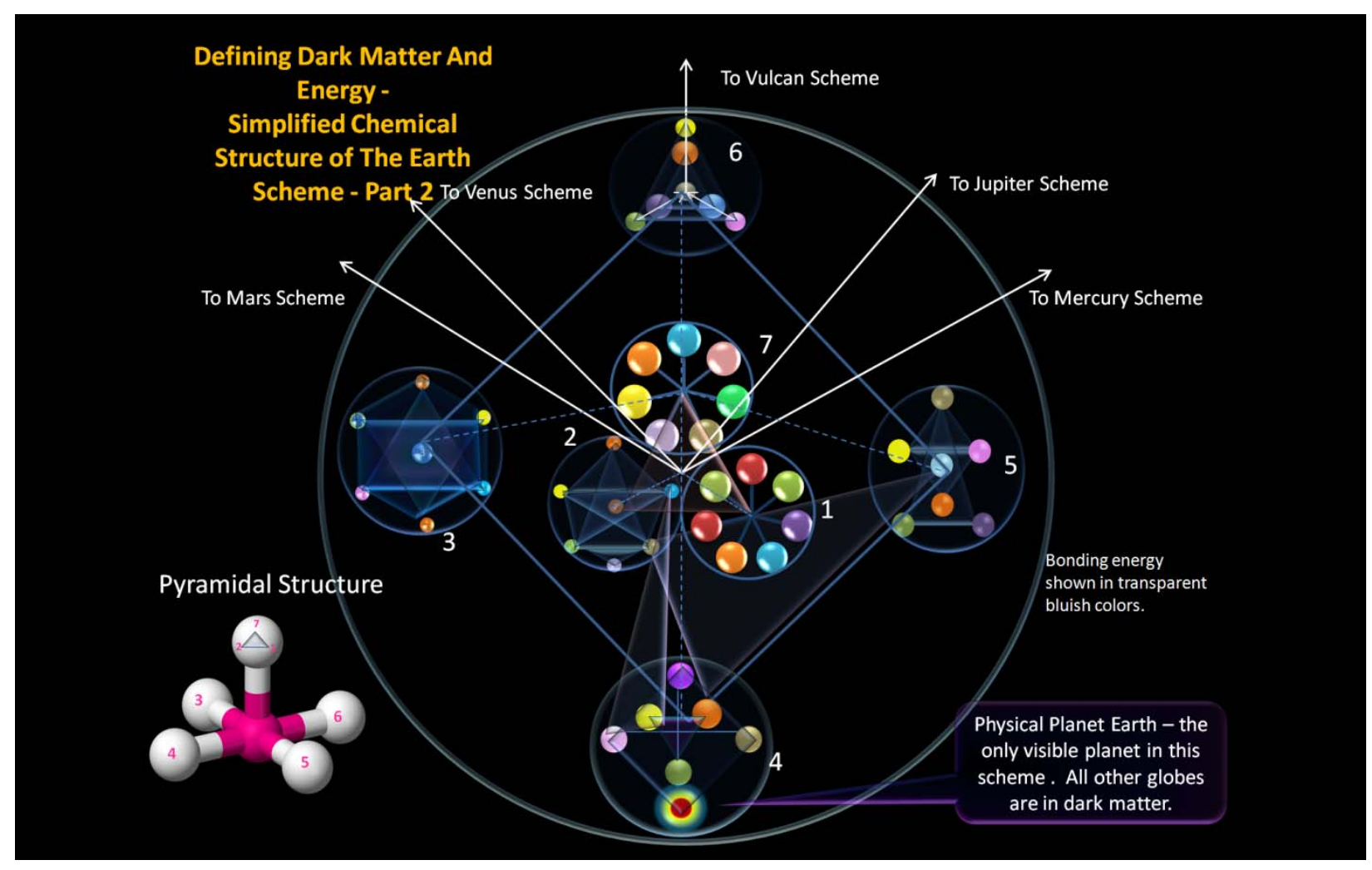

Figure 7. Defining Dark Matter and Energy - Chemical Structure of the Earth Scheme - Part 2.

All physical plane planets are the 'tip of the iceberg' and have chemical structures sitting in Dark Matter.

Two points to note from above:

1. The planes are numbered from 1 to 7 in reverse order of the major planes since the direction is below the axis. The planes are shown rotating east to west and exist in matter differentiated into seven qualities, grades or vibration.

2. The electromagnetic spectrum shown in planes 5 and 6 is analogous to the EM spectrum found in the $7^{\text {th }}$ plane and for the purpose of this hypotheses is defined as Dark Sound. It is phased to the plane. Fohat on these planes is referred to as Dark Energy.

\section{Everything Rotates}

If totusmateria is truly infinite in dimension then there will be an infinite number of universes coalescing, expanding and ceasing throughout all of creation. That is to say, our universe is but an atom in the fabric of infinity and that innumerable other universes exist simultaneously. Our universe is following the 6 step process and eventually will develop full rotary action with a well defined vortex at the center. Since this vortex has not been discovered it would suggest that the universe is somewhere around stage 3 - that is our universe is young. The WMAP Cold Spot (Rudnick, Brown, and Williams, 2007) MAY be the beginning of the well defined vortex. It should be noted that the black hole comes first, followed by the rotary action.

The rotation of atoms and our universe follows 3 stages. We are at stage 2 . 
1. Inertia - the initial stage as a result of inactivity and the relative quiescence of totusmateria.

2. Mobility - rotary movement begins as inertia is overcome by the incessant rotation of the black hole. The radiation of matter produces necessarily an effect upon other atoms in its environment, and this interaction and interplay causes repulsion and attraction according to polarity eventually producing cohesion of form. The implication here is that our universe is within the sphere of other 'universe-atoms' and that contact with the periphery of these universe-atoms in our universe's environment allows for the ingress and egress of force and heat. Evidence of this phenomenon is noted by Andrew Beckwith in a series of papers outlining the problem with entropy in the cosmos. (Beckwith, 2009a, 2009b, 2009c)

The actual complex structure of surrounding universe-atoms is not known but the simplest of the possible structures is analogous to deuterium $-{ }^{2} \mathrm{H}$.

3. Rhythm - this is the point of perfect balance.

Since the sound wave is dynamic and cyclic, the universe as we know it will continue to expand, and more rapidly as measurements indicate that the volume of the sound is increasing, but cessation will occur. It is important to note that everything rotates; light particles which rotate in reverse direction to the plane - west to east, planes which rotate east to west and energy which rotates north to south through the planes. The coordinates are illustrative only. Please see Figure 8.

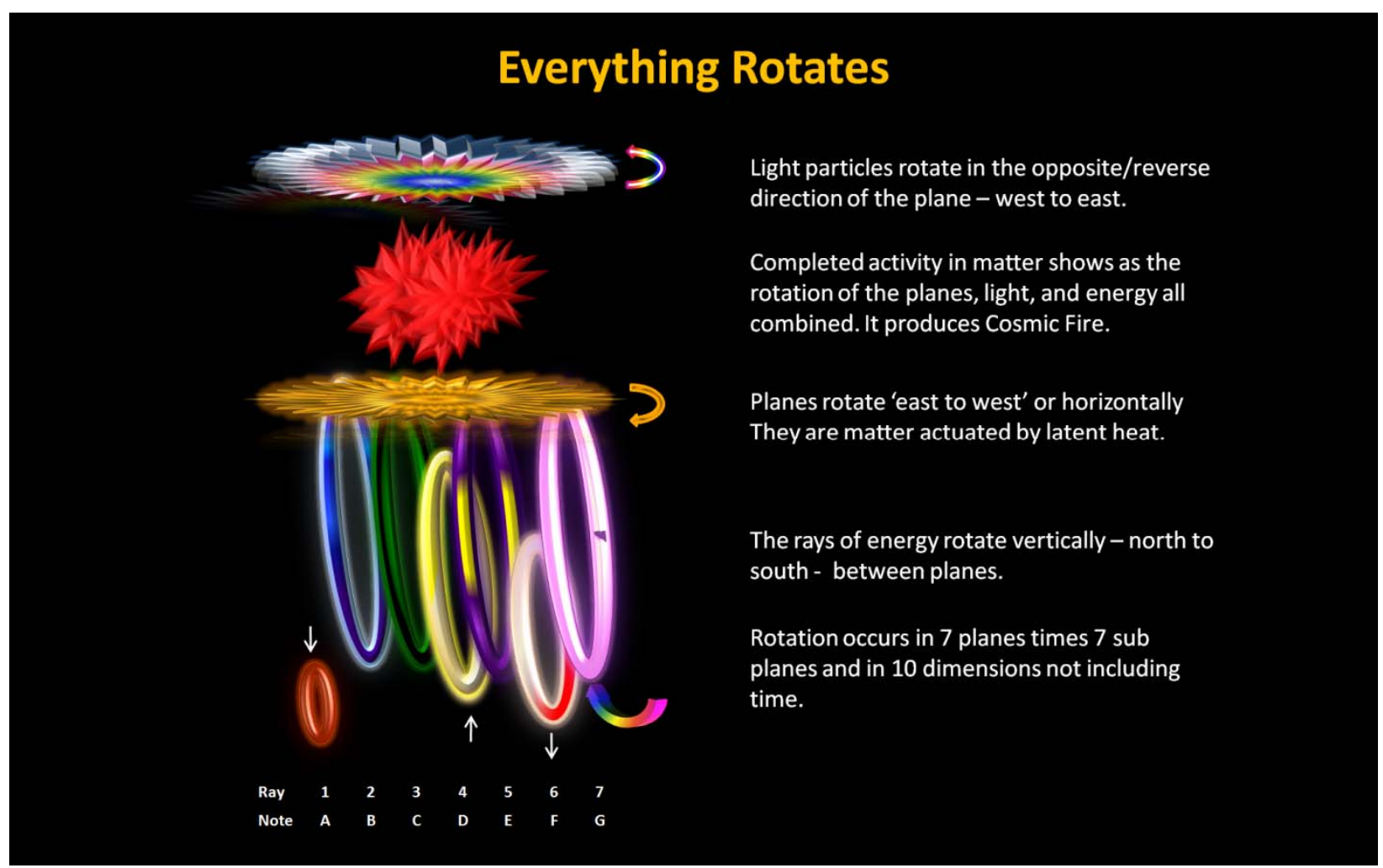

Figure 8. Everything Rotates.

The result of rotation produces:

1. Separation or the repulsive effect which allows for differentiation.

2. Momentum or the interior effect which keeps the planets at fixed points.

3. Friction or environal effect which keeps the atom vital and produces heat.

4. Absorption or the receptive or attractive effect which allows for the flow of force which animates the sphere. Further, as demonstrated in the planet Earth, each atom has a slight depression at the North Pole where the forces from without enters the sphere and counter rotates to the rotation of the sphere. From 
there the energy makes its way southward to a mid way point where it increases latent heat and produces added momentum. I am not referring to the Magnetic Dipole. The same is true for larger structures.

Cosmic fire is produced when planes, light and energy come together in a synchronous, stable environment an example being Betelgeuse which is in reality the energy center for a greater structure. Only animated atoms rotate. Those atoms such as the Earth's moon which have no light of their own, do not rotate and hence do not create internal heat are dead. In the case of the moon it is a cosmic carcass and scientific measurement should indicate disintegration. The position of the rays (not discussed in this paper) shown in Figure 8 is relative to the planet Earth at this time.

\section{The End - Big Bang}

The end for the universe is marked by a note as sound also destroys as well as build. Obscuration is signaled and disintegration of the form proceeds. The ovoid encapsulating our universe breaks down. As differentiated matter is merged with the root of matter, the three forms of electricity, positive, negative and synthesizing will unite bringing about spontaneous combustion. Force, energy and the production of heat cease and totusmateria returns to a quiescent state.

The conclusion here is that the 'Big Bang' is the end of manifestation - not the genesis.

All of the stages are clearly visible in the universe since solar systems and galaxies are atoms on a larger scale and are demonstrated in Figure 9.

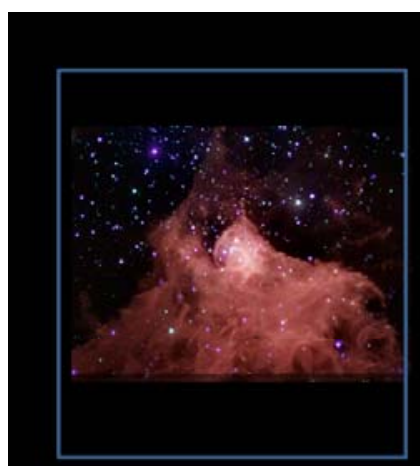

Step 1

Gas cloud

No rotation due to inertia

(a)

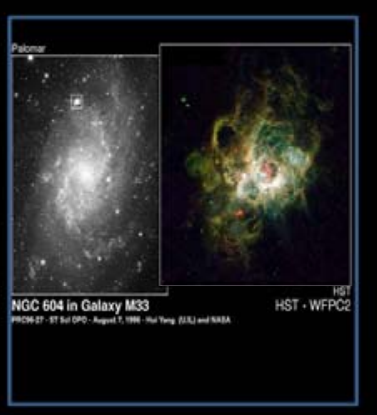

Step 2-4

Solidification with

mobility just noticeable (b)

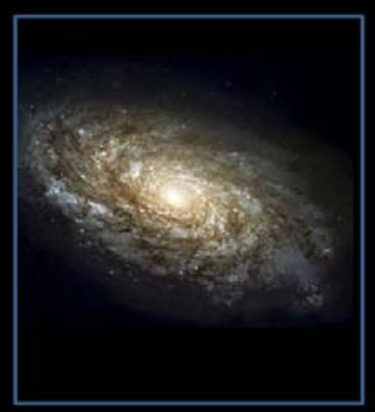

Step 5-6

Rotation and

rhythm

(c)

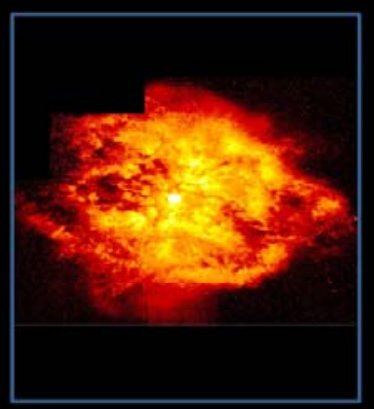

End-The Big Bang

for stars and the universe

Figure 9. These figures demonstrate the process, (a) shows the composite image of Cepheus B combines data from the Chandra X-ray Observatory and the Spitzer Space Telescope and demonstrates step 1; (b) shows Steps 2 to 4 which is shown by the nebula cataloged as NGC 604 is a giant star forming region, 1500 light years across, in the nearby spiral galaxy, M33. Over 200 newly formed, hot, massive, stars are scattered within a cavern-like, gaseous, interstellar cloud (see http://antwrp.gsfc.nasa.gov/apod/image/ngc604_hst_big.jpg); (c) shows Steps 5 and 6 which demonstrates a spiral galaxy, about 60 million light years from Earth as imaged by the Hubble Space Telescope. It is a fully formed cosmic atom with the characteristic black hole or vortex; (d) shows the final stage.

\section{Steps in the Universe Beginning to End}

To summarize the above, it is proposed that the life cycle of the universe follows these basic steps:

1. Totusmateria was present with no form, light or energy. 
2. A long radio wave penetrated totusmateria through the mechanism of a black hole. The frequency of the sound wave increased passing through microwave and infrared with the first real hint of birth appearing when the wavelength reached that of light. The planes developed and light appeared on all planes.

3. As the frequency increased it reached the hypergammic stage. The first atom to coalesce was hydrogen following the six stages. The cosmic zygote is encased in an ovoid.

4. Electrical energy in its 3 forms is functioning perfectly. The young universe is held together through the spheroidal form controlled by the synthesizing electrical energy of the second aspect.

5. Hydrogen converted to helium and the chemistry of space continued as more atoms coalesced out of totusmateria. Bach's Fugue becomes more complex, more black holes develop, differentiation begins and the volume increases. Expansion follows.

6. Following principles of recurrence relations and using the cosmic genetic code stored in totusmateria, planets, suns, stars and galaxies develop and proceed through the lifecycle in three basic stages: inertia, mobility and rhythm. The universe in its totality is influenced by other universe-atoms and rotates.

7. The end of the cycle is witnessed by a 'big bang'. Time ceases in the cycle, matter is reabsorbed back to the root and quiescence sets in.

\section{THE VACUUM OF SPACE AND GRAVITY}

Science assumes that space is nothing since they observe the vacuum although as outlined by Krauss (1999) in his book Quintessence, the void is actually thought to be Dark Matter with exotic properties. Aristotle also proclaimed there is no void. The vacuum has been unsatisfactorily explained as the near absence of matter due to the gravitational pull of the various large bodies occupying the space. However the hypothesis asserts that the observed gravity is a result of the attraction and repulsion of the nucleus and electrons of an atom/planet created by the rotary action and as mollified by a synthesizing electrical force maintaining equilibrium working through totusmateria; that is the second aspect of electricity to synthesize exerts forces on cosmic differentiated material both within the ovoid and externally. Therefore what the physicist calls gravity the chemist calls a bond. To demonstrate, Figure 10 gives the gravity and bonding structure relative to the planet Earth. Another planet would have a different structure. As shown below The Pleiades is the source for the neutral bonding energy which is found on the $4^{\text {th }}$ plane of the cosmic physical plane and in the $4^{\text {th }}$ dimension for visible globes. As a general rule blue/blue-white planets coalesce from totusmateria from the note of B and the energy of the second ray as noted in Figure 8 and typically carry the neutral form of electricity which binds.

The gravity or bonds affecting Earth follows:

1. A triangle of energy between Sirius, Alcyone which supplies the neutral bonding force, and the Sun. The bond between Sirius and our Sun is equivalent to J-coupling and the Nuclear Overhauser Effect magnetization transfer by dipolar coupling - both of which are prominent in the formation of DNA. Further, there is a solar union between ALL Suns which is not accounted for in the figure.

2. Our solar system has a positive polarity to The Pleiades.

3. Our solar system has a positive polarity to the combined forces of Merak, and Dubhe. The neutral bonding force comes from The Pleiades.

4. There are intermolecular forces between:

a. Our solar system, The Pleiades, and Merak (which forms a great cosmic triangle)

b. Mercury, Earth and Mars

c. Venus, Jupiter and Earth

d. Venus, Jupiter and Saturn.

5. The Sun is a great magnet and has a positive polarity to all of the planets, planetoids and other minor objects in the solar system.

6. The Earth has a positive polarity to Venus.

7. Dark Matter bonds are shown in Figure 7 


\section{(Simplified) Polarities and Bonds Relative to}

\section{Earth (only)}

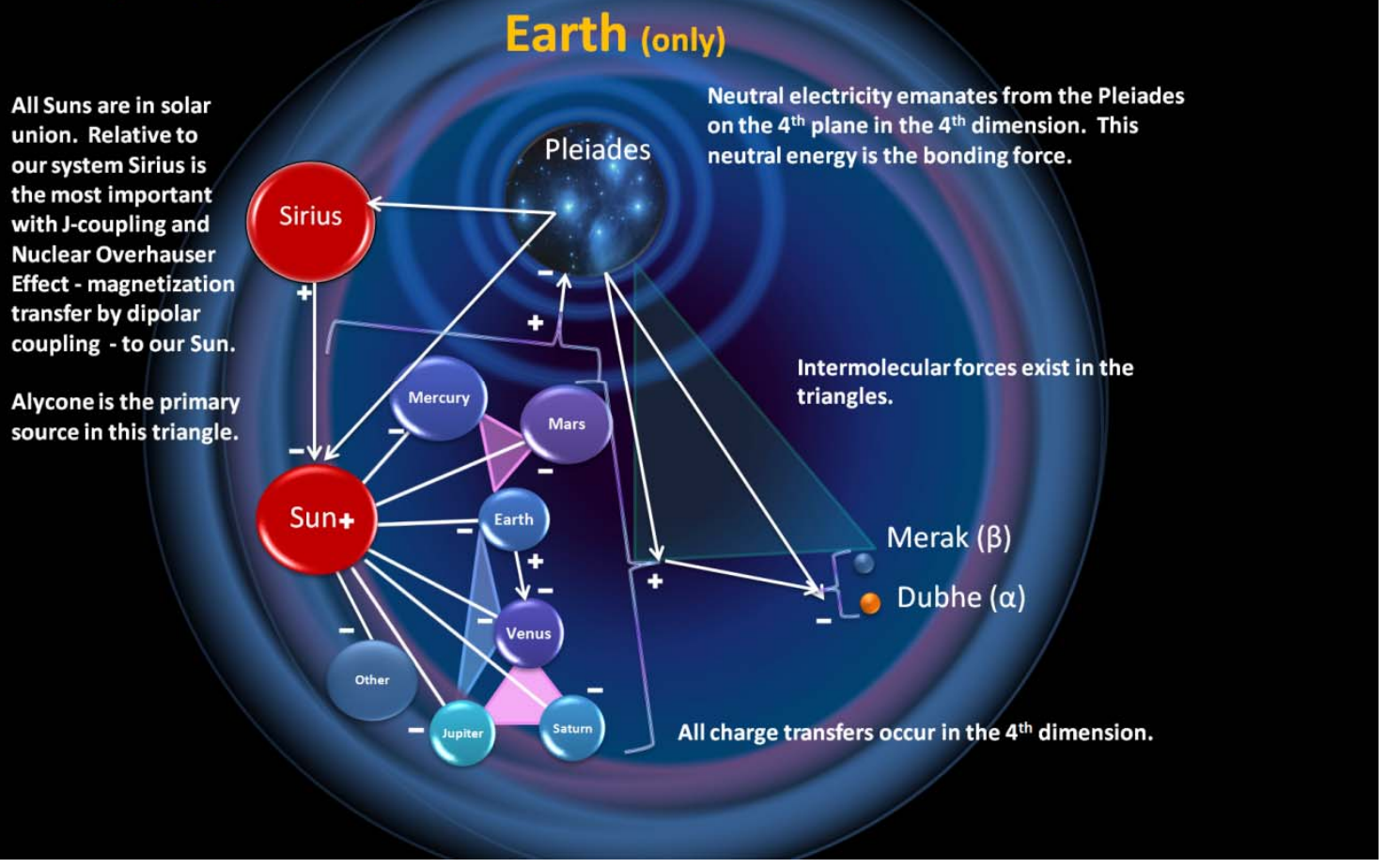

All Suns are in solar union. Relative to our system Sirius is with J-coupling and Nuclear Overhause magnetization fer by dipolar

Alycone is the primary source ir

Figure 10. Simplified Polarities and Bonds Relative to the Planet Earth

\section{Vacuum}

The issue with vacuum is that gravity is not the cause. The NOE Hypothesis states that the vacuum of space is an observed phenomenon created when sound at a certain frequency in the $5^{\text {th }}$ dimension is applied to totusmateria. The tone when phased to our atmosphere should allow for the reproduction of the phenomenon in the laboratory.

\section{WAVE PARTICLE DUALITY OF LIGHT}

The observed phenomenon of both wave and particle properties of light is explained in the hypothesis as the effect of light particles being bonded together with neutral electricity which gives the appearance of a wave under certain conditions. Light particles are matter of the finest order which is animated by fohat. The particle rotates in the opposite direction to the plane. It is bonded to the neighbouring particle through the $2^{\text {nd }}$ aspect of electricity neutral. This neutral form of electricity is NOT a particle and gives the appearance of a wave.

\section{SUMMARY}

In summary, the NOE Hypothesis states:

1. Totusmateria is the root of matter, the cosmic gene pool, in two basic forms: differentiated and undifferentiated.

2. Sound is defined as both known and dark. When the sound wave stops the form disintegrates and differentiated matter returns or is reabsorbed into the root of matter - totusmateria.

3. There are 7 planes with each plane further divided into 7 sub planes giving a total of 49 planes. The visible light spectrum replicates the 7 planes. 
4. There are 10 dimensions not including time. Bonds are found in the $4^{\text {th }}$ dimension, and the cosmic fugue is found in the $5^{\text {th }}$.

5. The atom coalesces out of totusmateria in a six step process.

6. Electricity comes in 3 parts - positive, negative and synthesizing - and is referred to as Fohat. Synthesizing, equilibrating or bonding energy comes from the $2^{\text {nd }}$ ray and typically manifests through blue/blue-white planets.

7. The origin of the universe was not created by a Big Bang, but a grand sound wave labeled Bach's Fugue which acted upon undifferentiated, primordial, pregenetic cosmic substance or totusmateria. The universe ends with a 'big bang'.

8. A Black Hole is the male aspect to cosmic reproduction and is a sound wave carrying fohat whirling at inconceivable rapidity.

9. Dark matter is a finer grade substance on a higher plane.

10. Everything rotates. Perfect rotation of matter, light and energy produces Cosmic Fire.

11. The known universe is but an atom in the fabric of infinity and is affected by other universe-atoms and will eventually be seen to rotate.

12. Modeling the universe needs to be completed using sound and classical mathematics, and color.

13. Gravity and chemical bonds are synonymous terms.

14. A vacuum is created by a sound wave and can be reproduced in the laboratory.

\section{CONCLUSIONS}

The purpose of this paper is to bridge esoteric philosophy with science and to resolve 'lost in translation' problems. The objective was to merge the two sciences and demonstrate that although the sources may be unusual the concepts appear to be valid. A coherent hypothesis for the origin and structure of the universe, Black Holes, Dark Matter, Dark Energy and resolving gravity is demonstrated. Validation however from the scientific community is necessary so the following next steps are suggested:

1. Map all of the Suns in the universe to determine the structure.

2. Assume Figure 10 is correct and calculate the gravity field for planet earth. Chemical bonding theory and the effect of magnetization of the SUN must be used. There should be good approximation. Precise calculations will require that all planets and schemes be included, plus rotation.

3. Complete entropy calculations from the early universe to the present assuming that the universe coalesced from totusmateria. Calculations must include pre-existing heat and force from other universe-atoms. It is suggested that initial calculations be modeled as deuterium in an octahedral structure.

4. Develop a lab experiment which demonstrates that a vacuum can be created by sound.

5. Greater study of the The Pleiades is required.

6. Mathematically define neutral electricity.

7. Develop lab experiments to discover the sound of light. It is suggested that the note of $\mathrm{G}$ and the color violet are synonymous and may be the easiest to find initially. Once the sound of light is determined then it will be possible to ascertain the tone of atoms, the chord of molecules and the tones of larger structures. A corollary to this is that the energy of the form will also be known. This will constitute a major paradigm shift in chemistry, physics and cosmology and will begin to unravel the Nature of Everything.

\section{DEFINITIONS}

Totusmateria - A material essence that is the fabric of space. It is the cosmic gene pool which allows for differentiation and replication. The medium that causes matter to condense out into existence in the presence of hypergammic radiation or sound waves. It is the sum total of all substances both differentiated and undifferentiated.

Hypergammic radiation - Wave energy that is at frequencies of Hydrogen (i.e. 2.27012x10 $0^{23} \mathrm{H}_{\mathrm{z}}$ ) or higher OR as phased to the plane it is on.

Bach's Fugue - The name given to the complex sound which controls all forms in the universe. The wave comes in 3 parts corresponding to the 3 types of electrical energies. 
Fohat - The name given to cosmic electricity in its triple nature and affects matter in all forms on all planes. It vitalizes all atoms.

\title{
ACRONYMS
}

\author{
EM - Electromagnetic NOE - Nature of Everything
}

\section{NOMENCLATURE}

\author{
$\mathrm{E}=$ energy in joules \\ $\mathrm{fm}=$ femtometres, $10^{-15}$ metres \\ $\mathrm{m}=$ mass in kilograms
}

\author{
$\mathrm{R}=$ radius of nucleus in femtometres \\ $\lambda=$ wavelength in metres \\ $v=$ frequency in Hertz
}

\footnotetext{
$\mathrm{A}=$ Atomic mass number, $1.660538782(83) \times 10^{-27} \mathrm{~kg}$

$\mathrm{c}=$ speed of light in metres per second $(\sim 299,792,458)$

$\mathrm{h}=$ Planck's constant $6.62606896(33) \times 10^{-34} \mathrm{Js}$

$\mathrm{r}^{0}=$ conversion factor of 1.2
}

\section{ACKNOWLEGDEMENT}

The author wishes to acknowledge Mark Garstin for his considerable contribution on the definition of hypergammic radiation and with great gratitude Dr. Andrew Beckwith for his dogged pursuit for something different from the Standard Model and his frequent encouragements that non physicists can contribute to the resolving of the cosmological problem.

\section{REFERENCES}

Bailey, Alice, “A Treatise on Cosmic Fire,” Fort Orange Press, Albany, N.Y., (1973).

Bailey, Alice, "A Treatise on The Seven Rays," Lucis Pub. Co, New York, (1962).

Beckwith, Andrew, "Entropy Growth in the Early Universe and Confirmation of Initial Big Bang Conditions," Numerical Analysis And Applied Mathematics: International Conference on Numerical Analysis and Applied Mathematics 2009, AIP Conference Proceedings, 1168, (2009), pp. 1077-1083.

Beckwith, Andrew, "Entropy Growth in the Early Universe, and the Search for Determining if Gravity is Classical or Quantum, Part I (Confirmation of Initial Big Bang Conditions?)," http://www.vixra.org/pdf/0909.0016v1.pdf, Sept, (2009a).

Beckwith, Andrew, "Entropy Growth in the Early Universe, and the Search for Determining if Gravity is Classical or Quantum, Part II (Do Physical Laws/ Physics Parameter Constants Remain Invariant from a Prior Universe, to the Present Universe?)," http://www.vixra.org/pdf/0909.0017v1.pdf, Sept, (2009b).

Beckwith, Andrew, "Entropy Growth in the Early Universe, and the Search for Determining if Gravity is Classical or Quantum , Part III (Is Gravity a Classical or Quantum Phenomenon at its Genesis 13.7 Billion Years Ago?)”; http://www.vixra.org/ pdf/0909.0010v2.pdf, Sept., (2009c).

Blavatsky, H. P., The Secret Doctrine: The Synthesis of Science, Religion, and Philosophy - Volume 1 Cosmogenesis, Pasadena, Calif.: Theosophical University Press, (1977).

Callender, Clifton, Quinn, Ian and Tymoczko, Dmitri, “Geometrical Music Theory,” Science 320(5874), 18 April, (2008), p. 346.

Krauss, Lawrence, Quintessence: The Search for Missing Mass in the Universe, Basic Books, New York, NY, (2000).

Nave, C. R., "HyperPhysics," CD, Department of Physics and Astronomy, Georgia State University, (2001).

Pedersen, Noralv, "Music is also Mathematics," Norwegian University of Science and Technology (NTNU), (2009).

Rudnick, L., Brown, S. and Williams, L. R., "Extragalactic Radio Sources and the WMAP Cold spot,” Astrophys.J., 671, (2007), pp. 40-44.

Thompson, D. J. and Fichtel, C. E., "Extragalactic gamma radiation - Use of galaxy counts as a galactic tracer," Astron. Ap., 109, (1982), p. 352.

Wrbanek, John D., Fralick, Gustave C. and Wrbanek, Susan Y., "Development of Techniques to Investigate Sonoluminescence as a Source of Energy Harvesting," in the proceeding of the 43rd Joint Propulsion Conference sponsored by the American Institute of Aeronautics and Astronautics, AIAA-2007-5596, Cincinnati, Ohio, July 8-11, (2007). 